\title{
A Smad3 and TTF-1/NKX2-1 complex regulates Smad4-independent gene expression
}

Kazunobu Isogaya ${ }^{1}$, Daizo Koinuma ${ }^{1}$, Shuichi Tsutsumi ${ }^{2}$, Roy-Akira Saito ${ }^{1,3}$, Keiji Miyazawa ${ }^{1,4}$, Hiroyuki Aburatani ${ }^{2}$, Kohei Miyazono ${ }^{1}$

${ }^{l}$ Department of Molecular Pathology, Graduate School of Medicine, The University of Tokyo, Bunkyo-ku, Tokyo 113-0033, Japan; ${ }^{2}$ Genome Science Division, Research Center for Advanced Science and Technology (RCAST), The University of Tokyo, Meguro-ku 153-8904, Japan; ${ }^{3}$ Department of Respiratory Medicine, Graduate School of Medicine, The University of Tokyo, Bunkyo-ku, Tokyo 113-0033, Japan; ${ }^{4}$ Department of Biochemistry, Interdisciplinary Graduate School of Medicine and Engineering, University of Yamanashi, 1110 Shimokato, Chuo, Yamanashi 409-3898, Japan

Thyroid transcription factor-1 (TTF-1, also known as NKX2-1) is a tissue-specific transcription factor in lung epithelial cells. Although TTF-1 inhibits the epithelial-to-mesenchymal transition induced by transforming growth factor- $\beta$ (TGF- $\beta$ ) in lung adenocarcinoma cells, the mechanism through which TTF-1 inhibits the functions of TGF- $\beta$ is unknown. Here we show that TTF-1 disrupts the nuclear Smad3-Smad4 complex without affecting the nuclear localization of phospho-Smad3. Genome-wide analysis by chromatin immunoprecipitation followed by sequencing revealed that TTF-1 colocalizes with Smad3 on chromatin and alters Smad3-binding patterns throughout the genome, while TTF-1 generally inhibits Smad4 binding to chromatin. Moreover, Smad3 binds to chromatin together with TTF-1, but not with Smad4, at some Smad3-binding regions when TGF- $\beta$ signaling is absent, and knockdown of Smad4 expression does not attenuate Smad3 binding in these regions. Thus, TTF-1 may compete with Smad4 for interaction with Smad3, and in the presence of TTF-1, Smad3 regulates the transcription of certain genes independently of Smad4. These findings provide a new model of regulation of TGF- $\beta$-Smad signaling by TTF-1.

Keywords: ChIP-seq; NKX2-1; Smad3; Smad4; TTF-1; TGF- $\beta$

Cell Research (2014) 24:994-1008. doi:10.1038/cr.2014.97; published online 25 July 2014

\section{Introduction}

Thyroid transcription factor-1 (TTF-1), also known as NKX2-1, is a tissue-specific homeodomain transcription factor expressed only in lung, bronchi, thyroid grand and forebrain. In the lung, TTF-1 is strongly expressed in type II alveolar cells and Clara cells, and regulates the expression of genes that encode surfactant proteins, such as SFTPB (encoding surfactant protein B). TTF-1 is thus thought to be the master regulator of lung epithelial differentiation [1].

TTF-1 is expressed in $75 \%-80 \%$ of lung adenocar-

Correspondence: Kohei Miyazono ${ }^{a}$, Daizo Koinuma

${ }^{\mathrm{a}, \mathrm{b}}$ Tel: +81-3-5841-3345; Fax: +81-3-5841-3354

aE-mail: miyazono@m.u-tokyo.ac.jp

bE-mail: koinuma@m.u-tokyo.ac.jp

Received 21 November 2013; revised 6 April 2014; accepted 4 May 2014; published online 25 July 2014 cinoma patients $[2,3]$. Among patients with lung adenocarcinoma, those with TTF-1-positive cancer exhibit better prognosis than those with TTF1-negative cancer [4-6]. Using a transgenic lung cancer mouse model, deletion of TTF-1 has been shown to promote invasion and metastasis of lung adenocarcinoma, in part due to the role of TTF-1 in HMGA2 expression [7]. TTF-1 was also shown to reduce cell motility and metastasis through induction of $M Y B P H$ expression [8]. These findings strongly suggest that TTF-1 functions as a tumor suppressor in lung adenocarcinoma. In contrast, genomics analyses revealed that human TTF1/NKX2-1 gene was amplified in 10\%$15 \%$ of lung adenocarcinomas; it is therefore called a lineage-survival oncogene [9-12]. TTF-1 has been shown to exhibit a pro-survival effect by inducing ROR1 expression, which enhances AKT signaling through the EGFErbB3-PI3 kinase axis [13]. It has also recently been reported that amplified TTF-1 and FOXA1 cooperatively regulate expression of the $L M O 3$ oncogene, which me- 
diates cell survival downstream of TTF-1 [14]. Although the precise mechanism(s) of favorable prognosis brought by TTF-1 remains unknown, it is possible that TTF-1 interacts with other transcription factors and alters their signaling activities.

Transforming growth factor- $\beta$ (TGF- $\beta$ ) is a multifunctional cytokine with bidirectional roles in cancer progression $[15,16]$. TGF- $\beta$ binds to type II and type I receptors, resulting in phosphorylation of the receptor-regulated Smads (R-Smads): Smad2 and Smad3. R-Smads form hetero-oligomeric complexes with Smad4, and translocate into the nucleus $[15,16]$, where they regulate the transcription of target genes through interaction with other transcription factors. Smad3 and Smad4 bind directly to chromatin through their N-terminal MH1 domains; Smad2 does not bind directly to chromatin because of an insert sequence that is present in its MH1 domain [17, 18]. Several groups have reported genome-wide analyses of the binding patterns of TGF- $\beta$ receptor-regulated Smads in various cancer cell lines and embryonic stem cell-derived cells [19-25]. These results reveal varied Smad-binding profiles in different cell types, indicating that "cell-specific context" is important for the response to TGF- $\beta$ signaling.

Smad3 reportedly regulates the transcriptional activity of TTF-1 [26, 27]. We previously reported that TTF-1 inhibits TGF- $\beta$-induced epithelial-to-mesenchymal transition (EMT) in lung adenocarcinoma cells [28]. Conversely, TGF- $\beta$ decreased endogenous expression of TTF- 1 [28]. Thus, functional links between TTF-1 and TGF- $\beta$ signaling appear to be important for the progression of lung adenocarcinoma. Genome-wide analyses of TTF-1 binding have recently been reported [14, 29]; however, how TTF- 1 regulates TGF- $\beta$-Smad signaling remains to be elucidated. Here, we identified and compared Smad3-, Smad4- and TTF-1-binding sites in the H441 lung adenocarcinoma cell line to understand the mechanism by which TTF- 1 inhibits TGF- $\beta$ signaling. Our data suggest that TTF- 1 regulates TGF- $\beta$-Smad signaling by competing with Smad4, and that Smad3 acts together with TTF1 to regulate expression of certain genes, e.g., LMO3, in a Smad4-independent manner. TTF-1 may therefore contribute to context-dependent regulation of TGF- $\beta$ and Smad3 signaling in lung epithelial cells and lung adenocarcinoma cells.

\section{Results}

\section{TTF-1 disrupts the Smad3-Smad4 complex}

First, we examined whether TTF-1 affects the complex formation of Smad3 with Smad4, as well as the phosphorylation and nuclear translocation of Smad3.
TTF-1 was exogenously expressed in A549 cells, which lack the expression of endogenous TTF-1, with an adenoviral vector AdTTF-1. Co-immunoprecipitation assay demonstrated that TTF-1 bound to Smad3 with or without TGF- $\beta$ stimulation, and formation of the Smad 3 and Smad4 (Smad3-Smad4) complex induced by TGF- $\beta$ was strongly inhibited by TTF-1 overexpression (Figure 1A). Next, we fractionated the nucleus and cytoplasm of A549 cells infected with AdTTF-1, and detected phosphorylated Smad3 (pSmad3) by immunoblotting. pSmad3 was detected in the nucleus upon TGF- $\beta$ stimulation, while TTF-1 was located mainly in the nucleus in the presence or absence of TGF- $\beta$ stimulation (Figure 1B). TTF-1 overexpression did not suppress either phosphorylation of Smad3 or nuclear translocation of Smad3 and Smad4.

We also confirmed the localization of Smad3 and Smad2 using an in situ proximity ligation assay (in situ PLA). In agreement with the findings of the subcellular fractionation experiments (Figure 1B), forced expression of TTF- 1 did not affect TGF- $\beta$-induced nuclear translocation of Smad3 and Smad2 (Supplementary information, Figure S1A). By using anti-TTF-1 and anti-Smad3 antibodies, we found that TTF-1 was located in the vicinity of Smad3 in the nucleus with or without TGF- $\beta$ stimulation (Supplementary information, Figure S1B).

Next, we assessed formation of the Smad3-Smad4 complex by in situ PLA (Figure 1C). The Smad3-Smad4 complex was observed in both the nucleus and the cytoplasm in A549 cells infected with a control adenovirus (AdLacZ), and most of the nuclear Smad3-Smad4 complex disappeared in the presence of TTF-1. The nuclear complex containing Smad2 and Smad4 was also decreased by TTF-1 overexpression (data not shown). We then performed in situ PLA assays using H441 human lung adenocarcinoma cells, which endogenously express TTF-1 [28]. Knockdown of TTF-1 by siRNA treatment (Supplementary information, Figure S4A) increased the number of nuclear Smad3-Smad4 complexes in H441 cells (Figure 1D), suggesting that TTF-1 disrupts the Smad3-Smad4 complex in the nucleus.

\section{TTF-1 inhibits Smad3 binding to chromatin}

TTF-1 has been reported to inhibit certain TGF- $\beta$ Smad3 signaling pathways [28]; therefore, it is possible that Smad3 is present in the nucleus but fails to bind to target genomic regions in the presence of TTF1. Thus, we performed chromatin immunoprecipitation (ChIP)-quantitative PCR (qPCR) analysis using a Smad3-specific antibody. Exogenous expression of TTF1 strongly inhibited the binding of Smad3 to the plasminogen activator inhibitor-1 (PAI-1, also known as SER$P I N E 1)$ and SMAD7 promoters (Figure 1E). Next, we 
A

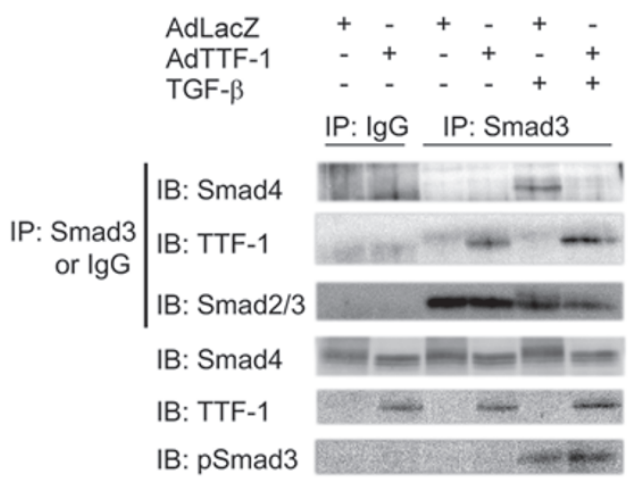

C
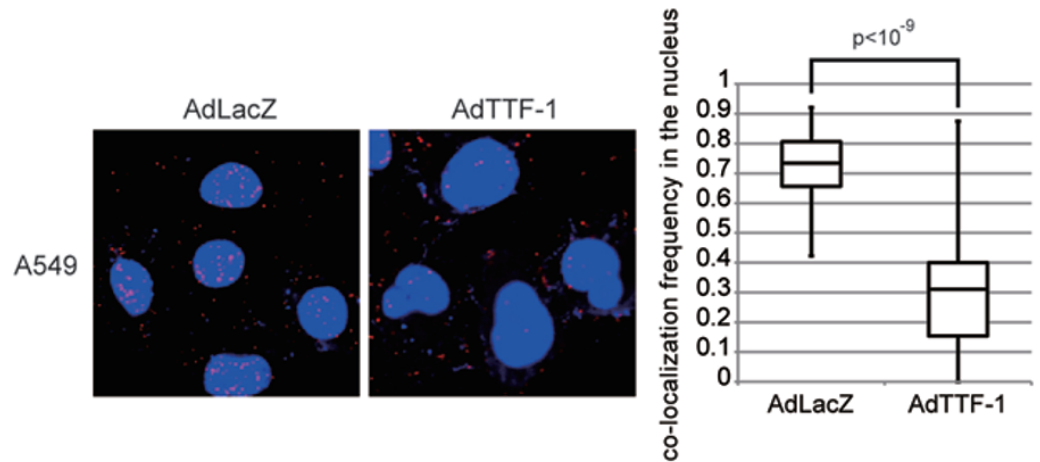

$\mathrm{D}$

B
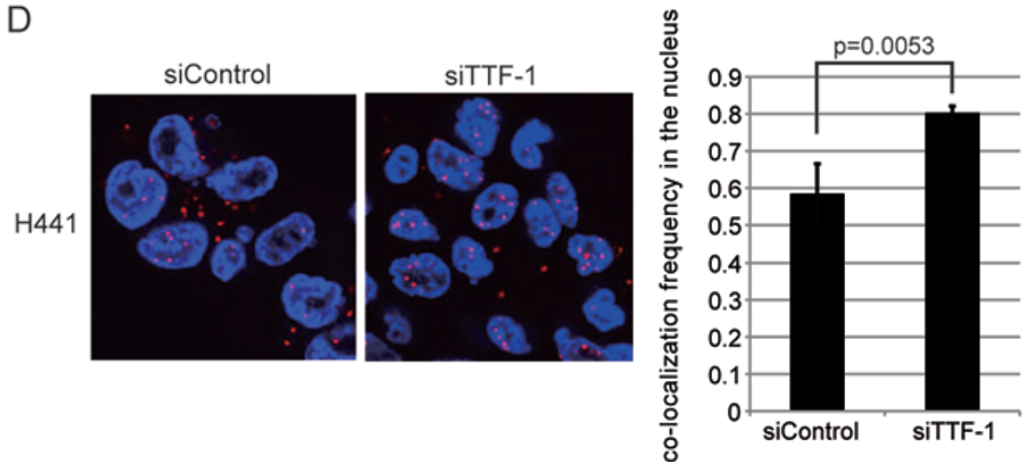

$\mathrm{F}$
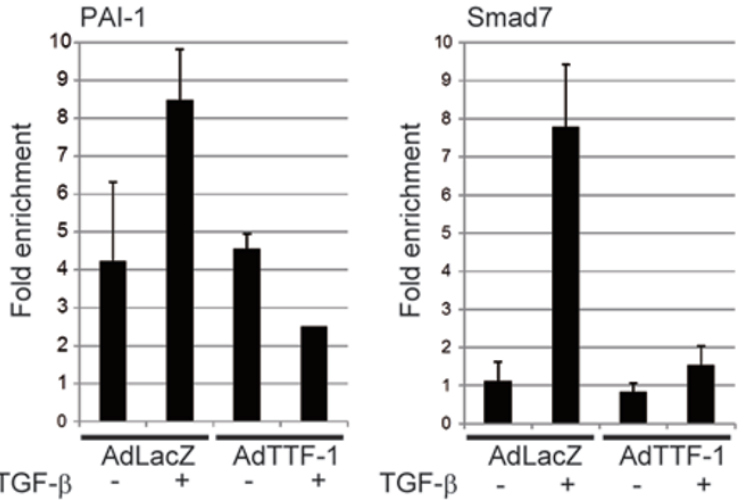

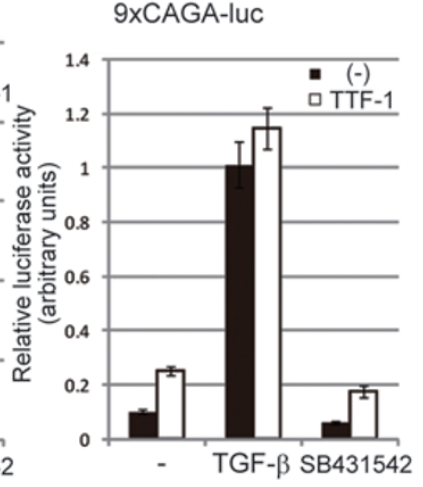

Figure 1 TTF-1 interacts with Smad3 independently of TGF- $\beta$ and inhibits Smad3-Smad4-induced transcription of target genes. (A) A549 cells were infected with the adenoviral TTF-1 expression vector (AdTTF-1) or control adenovirus (AdLacZ) as indicated. At $24 \mathrm{~h}$ post-infection, cells were treated with TGF- $\beta$ for $1.5 \mathrm{~h}$. Cell lysates were immunoprecipitated (IP) with anti-Smad3 antibody or control mouse IgG, and Smad3-bound TTF-1 and Smad4 were detected by immunoblotting (IB). (B) A549 cells were infected with adenoviral vectors as in A. Nucleic and cytoplasmic fractions of the cells were obtained $2 \mathrm{~h}$ after TGF- $\beta$ stimulation, and the levels of phosphorylated Smad3 (pSmad3), total Smad3 (tSmad3) and Smad4 were detected by IB. (C, D) A549 cells infected with the indicated adenoviral vectors (AdLacZ or AdTTF-1) (C) and H441 cells transfected with control siRNA (siControl) or TTF-1 siRNA (siTTF-1) (D) were treated with TGF- $\beta$ for $1.5 \mathrm{~h}$, and the Smad3-Smad4 complex was detected using anti-Smad3 and anti-Smad4 antibodies. PLA signals (red) detected in the nuclei (blue) of A549 cells infected with AdLacZ or AdTTF-1 or H441 cells treated with siControl or siTTF-1 were counted (right panels). (E) A549 cells were infected with adenoviral TTF-1 or LacZ expression vectors and treated with TGF- $\beta$ for $1.5 \mathrm{~h}$. Then, a ChIP assay using Smad3-specific antibody was performed to analyze the binding of Smad3 to the PAl-1 and SMAD7 promoter regions. (F) A549 cells were transfected with the TTF-1-expression vector or control vector and treated with TGF- $\beta$ for 12 h or SB431542 for 12 h. Luciferase reporter assays using PAI-1-luc containing PAI-1 natural promoter or 9× CAGA-luc containing tandemly repeated SBEs were performed. 
examined the effects of TTF- 1 on TGF- $\beta$-induced transcription using a luciferase reporter containing the PAI-1 promoter (PAI-1-luc) and a reporter containing tandemly repeated Smad-binding elements (SBEs; 9× CAGA-luc). TTF-1 overexpression inhibited the promoter activity of PAI-1-luc induced by TGF- $\beta$, but did not affect that of $9 \times$ CAGA-luc (Figure 1F). However, treatment with the TGF- $\beta$ type I receptor inhibitor SB431542 effectively reduced TGF- $\beta$-induced activation of both reporters (Figure $1 F)$. These findings suggest that certain cis-regulatory element(s) other than the canonical SBE might be required for TTF-1 to inhibit Smad-induced transcription.

Genome-wide identification of Smad3-, Smad4- and TTF1-binding regions in $\mathrm{H} 441$ cells

Next, we performed ChIP-seq analyses using H441 cells transfected with siRNA for TTF-1 (siTTF-1) or control siRNA (siControl), and stimulated the cells with TGF- $\beta$. Using a false discovery ratio (FDR) cut-off of 0.01 , we have identified 8941 and 14145 Smad3-binding regions from the ChIP-seq data of TGF- $\beta$-treated H441 cells transfected with control and TTF-1 siRNAs, respectively. ChIP-seq data from TGF- $\beta$-stimulated H441 cells with and without TTF-1 siRNA yielded 1605 and 1 Smad4-binding peaks, respectively, with a FDR cutoff of 0.1; the same sample set also yielded 21292 and 31083 TTF-1-binding peaks with and without TGF- $\beta$ stimulation, respectively, with a FDR cutoff of 0.01 (Supplementary information, Tables S1-S6).

There were several significant Smad3- and Smad4-binding peaks in the promoter region of PAI-1 gene, as previously reported [25] (see Figure 2D). The enrichment of Smad3 in the PAI-1 promoter region was validated by ChIP-qPCR (Supplementary information, Figure S2). There were also significant TTF-1-binding peaks in the promoter region of SFTPB gene [14] (Supplementary information, Figure $\mathrm{S} 3 \mathrm{~A}$ and $\mathrm{S} 3 \mathrm{~B}$ ). We performed de novo motif prediction in the TTF-1 ChIP-seq data using the CisGenome Gibbs motif sampler. One of the obtained motifs (Supplementary information, Figure S3C) was very similar to the TTF-1-binding motif recently reported by others [14], indicating that the ChIP-seq analysis appeared to successfully recognize binding regions of TTF-1.

Binding of Smad3 to chromatin at the Smad3-only regions is mainly affected by TTF-1

We compared the identified Smad3-, Smad4- and TTF-1-binding peaks obtained by the ChIP-seq analyses of H441 cells. Approximately 80\% (7 201/8 941) of the Smad3-binding peaks in H441 cells treated with siControl were also observed in cells treated with siTTF-1 (Figure 2A-i). When transfected with TTF-1 siRNA, approximately 75\% (1 281/1 605) of the Smad4-binding peaks were common to the Smad3-binding peaks (Figure 2A-ii). It should be noted that the number of binding sites of each transcription factor depends on the efficiency of antibodies used for ChIP analysis, and that the ChIP efficiency of the anti-Smad4 antibody was much lower than that of the anti-Smad3 antibody. More than $85 \%$ (7 839/8 941) of the Smad3-binding peaks were common to the TTF-1-binding peaks (Figure 2B-i). Knockdown of TTF-1 increased the number of Smad3-binding peaks, but most of the new peaks were not common to TTF-1-binding peaks (Figure 2B-ii). About one-half (797/1 605) of the Smad4-binding peaks in siTTF-1-transfected cells were common to TTF-1binding peaks (Figure 2B-iii).

To analyze these data quantitatively, we obtained a read count of each Smad3-binding peak from the ChIPseq data of Smad3 in H441 cells treated with siTTF-1 and siControl. We then calculated the siControl/siTTF-1 ratio of the read counts at each binding peak. If this ratio is high, then TTF-1 does not tend to inhibit Smad3 binding in the respective region. If the ratio is low, the binding strength of Smad3 in the respective region is likely to be attenuated by the presence of TTF-1. We arranged the Smad3-binding peaks with respect to these ratios, chose the top 2000 and bottom 2011 peaks and examined whether there were any TTF-1-binding region(s) within $200 \mathrm{bps}$ of the peak summit. Interestingly, more than $80 \%$ of the top 2000 binding peaks have TTF-1-binding regions within $200 \mathrm{bps}$ of their binding summits, while TTF-1 binds to only $8 \%$ of the bottom 2011 peaks (Figure $2 \mathrm{C})$. Therefore, Smad3 colocalizes with TTF-1 on chromatin in the presence of TTF-1, and the inhibitory effect of TTF-1 on Smad3 does not appear to be induced by competition in chromatin binding.

Analysis of the ChIP-seq data revealed two types of Smad3-binding regions, i.e., Smad3-TTF-1 common regions and Smad3-only regions, even around a single target gene locus. For example, there are seven Smad3-binding peaks around the PAI-1 gene; two of them are Smad3-TTF-1 common regions (Figure 2D, peaks $f$ and $g$ ) and five are Smad3-only regions (Figure $2 \mathrm{D}$, peaks a-e). We performed sequential anti-Smad3 and anti-Smad4 ChIP-qPCR at the peak e in Figure 2D. We found that Smad3 and Smad4 were colocalized at this peak, and that the binding strength was increased when TTF-1 was knocked down (Figure 2E). Next, we used scatter plotting to compare the changes in read counts of Smad3-TTF-1 common regions (Figure 2F, upper panel) or Smad3-only regions (Figure 2F, lower panel) between siControl- and siTTF-1-transfected cells. The read counts in most of the Smad3-TTF-1 common regions did not 


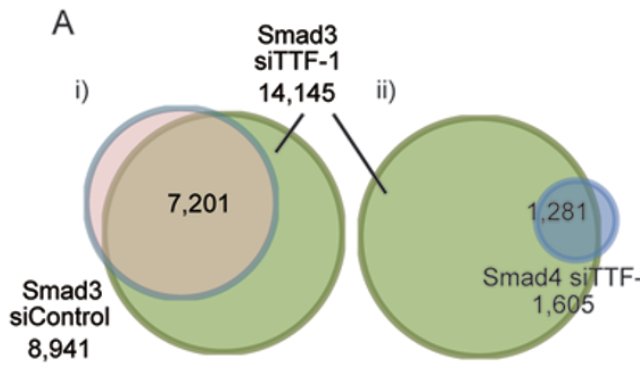

C
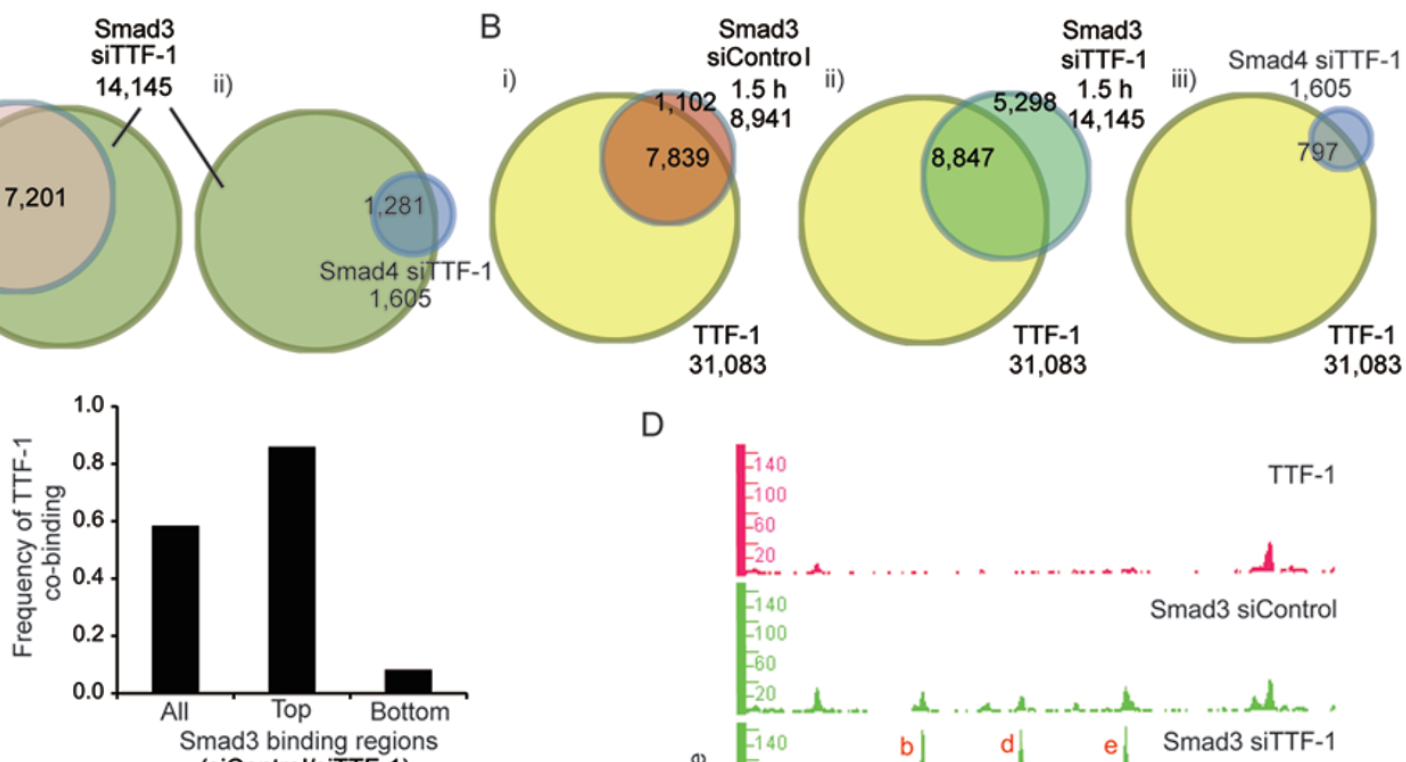

E (siControl/siTTF-1)

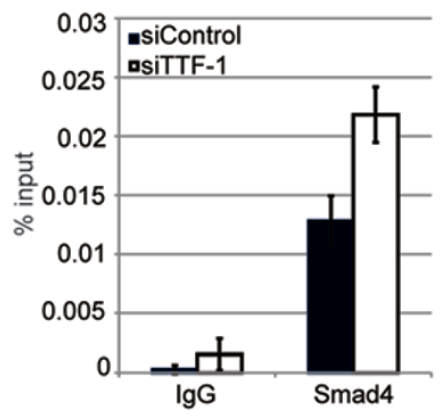

D

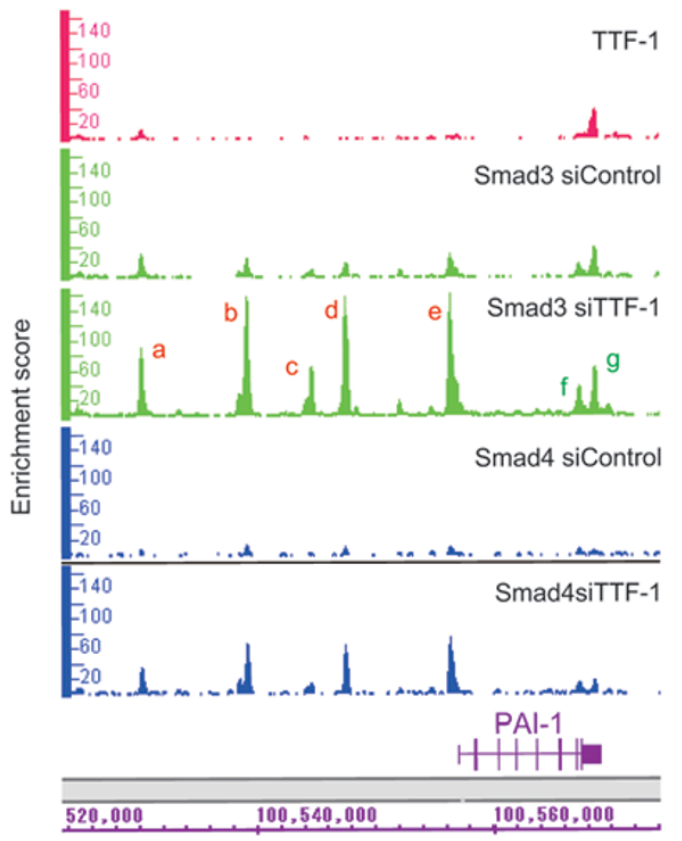

$\mathrm{F}$

Common binding regions

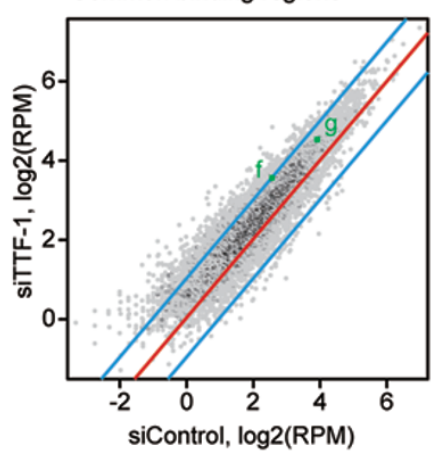

Smad3-only regions

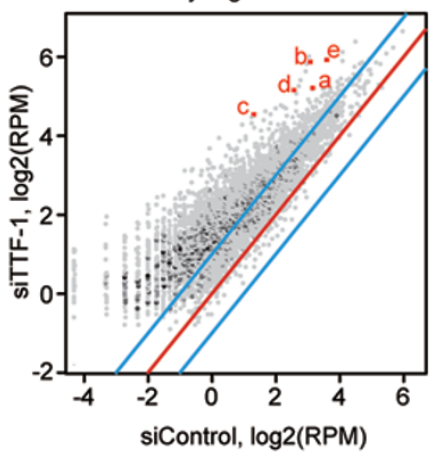

G

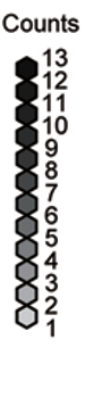

Counts

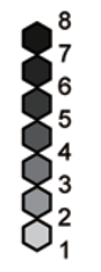

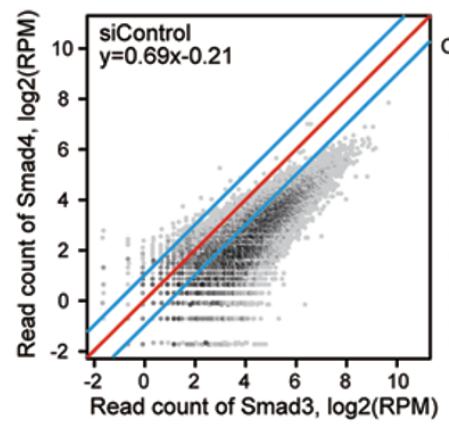

$\mathrm{H}$
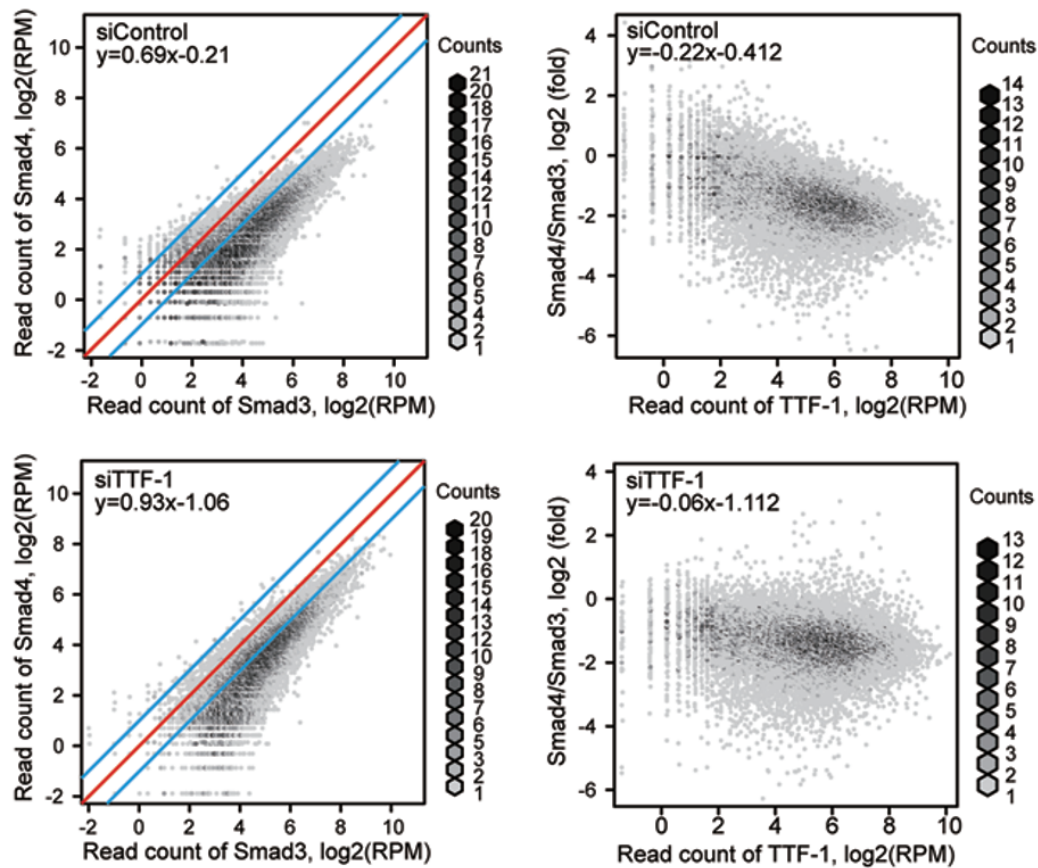
Figure 2 ChIP-seq analysis reveals colocalization of Smad3 and TTF-1, and competition of Smad4 and TTF-1 on chromatin in H441 cells. (A, B) Comparison of the numbers of Smad3-, Smad4- and TTF-1-binding regions in H441 cells obtained from ChIP-seq analyses. (A) i: Pink and green circles represent Smad3-binding regions in H441 cells treated with TGF- $\beta$ for $1.5 \mathrm{~h}$ with control and TTF-1 siRNA, respectively (FDR $=0.01)$. ii: Blue circle represents Smad4-binding regions in siTTF1-transfected H441 cells treated with TGF- $\beta$ (FDR $=0.1)$. (B) Yellow circle represents TTF-1-binding regions in H441 cells without TGF- $\beta$ stimulation. Orange (i) and green (ii) circles represent Smad3-binding regions in siControl- and siTTF-1-transfected H441 cells treated with TGF- $\beta$, respectively, and the blue circle (iii) represents the Smad4-binding regions as shown in A-ii. (C) The frequency of TTF-1-binding at the Smad3-binding sites. Smad3-binding strength at each binding region was defined as read counts normalized by total mapped reads of the dataset (14 145 Smad3-binding peaks determined in H441 cells transfected with siTTF-1). The read count ratio of each binding region was obtained by calculating the normalized read counts of siControl/siTTF-1. The frequency of Smad3-binding sites common to TTF-1 was calculated in two groups: the top 2000 and bottom 2011 regions with high and low siControl/siTTF-1 ratios, respectively. (D) TTF-1-, Smad3- and Smad4-binding regions in the PAl-1 gene locus. Peaks a-e indicate Smad3-only regions; peaks $f$ and $g$ indicate Smad3-TTF-1 common regions. (E) ChIP-reChIP analysis of Smad3-Smad4 co-binding to DNA in H441 cells. Cells were stimulated with TGF- $\beta$ for $1.5 \mathrm{~h}$ and fixed. Samples were eluted after anti-Smad3 ChIP, followed by secondary ChIP as indicated (IgG or anti-Smad4). Primers specific for the Smad3-binding region (peak e in Figure 2D) were used for evaluation by real-time PCR. (F) Read counts of Smad3-binding strength in the Smad3-TTF-1 common binding regions (upper panel) and the Smad3-only binding regions (lower panel) were obtained, and comparison was made in each region in TGF- $\beta$-treated H441 cells transfected with siControl and siTTF-1. Red dots (a-e) and green dots ( $f$ and $g$ ) indicate the binding regions shown in $\mathbf{D}$. (G) Read counts in all Smad3-binding regions were obtained from the Smad3-ChIP-seq and Smad4-ChIP-seq data of TGF- $\beta$-treated H441 cells transfected with siControl (upper panel) or siTTF-1 (lower panel), and read counts of Smad3 and Smad4 were compared in each region. (H) Binding strengths of TTF-1 were determined as normalized read counts of TTF-1 and compared with the relative read counts' ratios of Smad4/Smad3 in the Smad3-binding sites in H441 cells transfected with siControl (upper panel) or siTTF-1 (lower panel). RPM, reads per million mapped reads.

increase more than two-fold in H441 cells with siTTF-1 compared with cells with siControl, while the read counts in the Smad3-only regions increased more than two-fold in the siTTF-1-treated cells. Taken together, these findings suggest that TTF-1 mainly inhibits Smad3 binding in Smad3-only regions.

\section{Inhibitory effect of TTF-1 on the binding of Smad4 to chromatin}

We obtained and analyzed read counts of Smad4 binding in all of the Smad3-binding regions of H441 cells with siTTF-1 from the Smad4 ChIP-seq data. Then, we compared the binding strengths of Smad 3 and Smad4 in each Smad3-binding peak, and analyzed them by scatter plotting (Figure 2G). Regardless of the presence of siControl and siTTF-1, the binding strengths of Smad3 and Smad4 were strongly correlated. However, the slope of the regression curve was sharper for cells treated with siTTF-1 than for cells treated with siControl. Thus, the binding strength of Smad4 may be weaker in the presence of TTF-1 than in its absence, especially where strong binding peaks of Smad3 were observed.

As TTF-1 appeared to affect Smad4-chromatin binding more strongly than Smad3-chromatin binding, we obtained read counts of TTF- 1 in the absence of TGF- $\beta$ treatment in the Smad3-binding regions shown in Figure $2 \mathrm{G}$ (upper panel), and compared them with the relative binding strengths of Smad3 and Smad4 by calculating the read count ratios of Smad4/Smad3 (Figure 2H).
Smad4/Smad3 read count ratios diminished in peaks where TTF-1 strongly bound to chromatin together with Smad3 in H441 cells treated with siControl. In contrast, there is no correlation between TTF-1-binding strengths and Smad4/Smad3 read count ratios observed in siTTF-1-treated cells. In such case Smad4 bound to chromatin equally well, regardless of whether the chromatin regions represent Smad3-TTF-1 common regions or Smad3-only regions (data not shown). Therefore, TTF-1 may compete with Smad4 to bind to Smad3.

\section{TTF-1 suppresses the expression of EMT-related genes and regulates that of apoptosis-related genes}

We obtained expression array data from H441 cells with siTTF-1 or siControl in the presence and absence TGF- $\beta$ stimulation. The knockdown efficiency of TTF1 by siRNA was confirmed (Supplementary information, Figure S4A). We chose 1049 genes that were expressed in H441 cells and induced by TGF- $\beta$ stimulation. We compared changes in mRNA expression levels of these genes and Smad3-binding signals in their promoter regions between cells transfected with siControl and siTTF-1, and found that elevation of Smad3-binding signals was correlated with the upregulation of gene expression in siTTF-transfected cells (Figure 3A). Representative genes, whose expression was induced by TGF- $\beta$ and enhanced more than two-fold by knockdown of TTF-1, are shown in Supplementary information, Table S7.

We next performed ontology analysis of the microar- 
A

$\log 2-1$

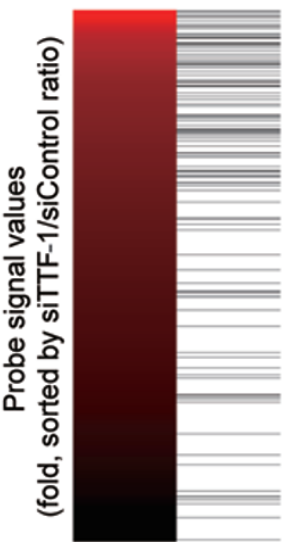

siTTF-1/ Elevated siControl Smad3

binding

B

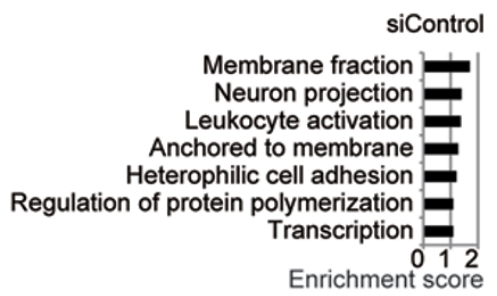

C

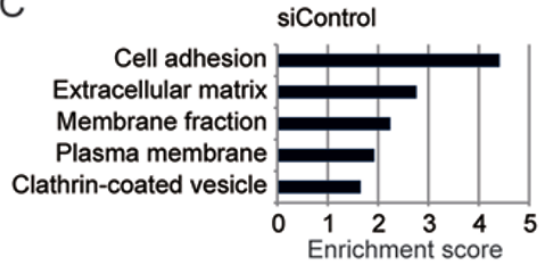

siTTF-1

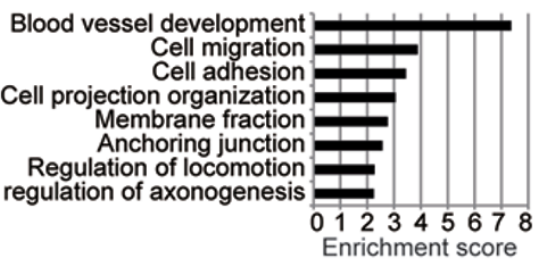

siTTF-1

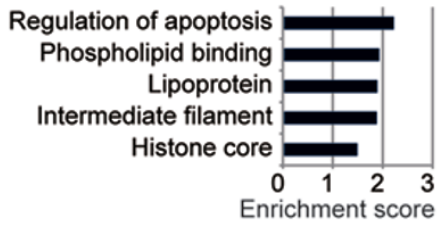

Assembled

Independent
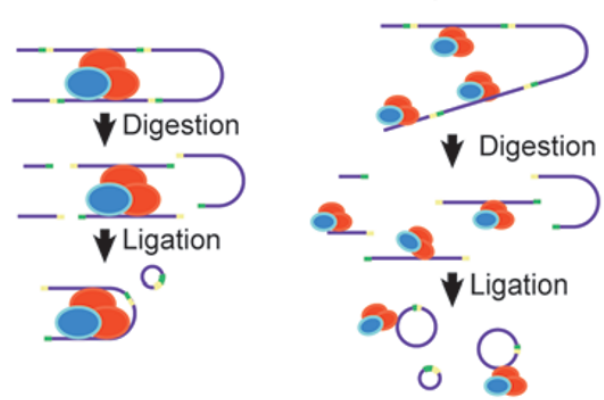

E
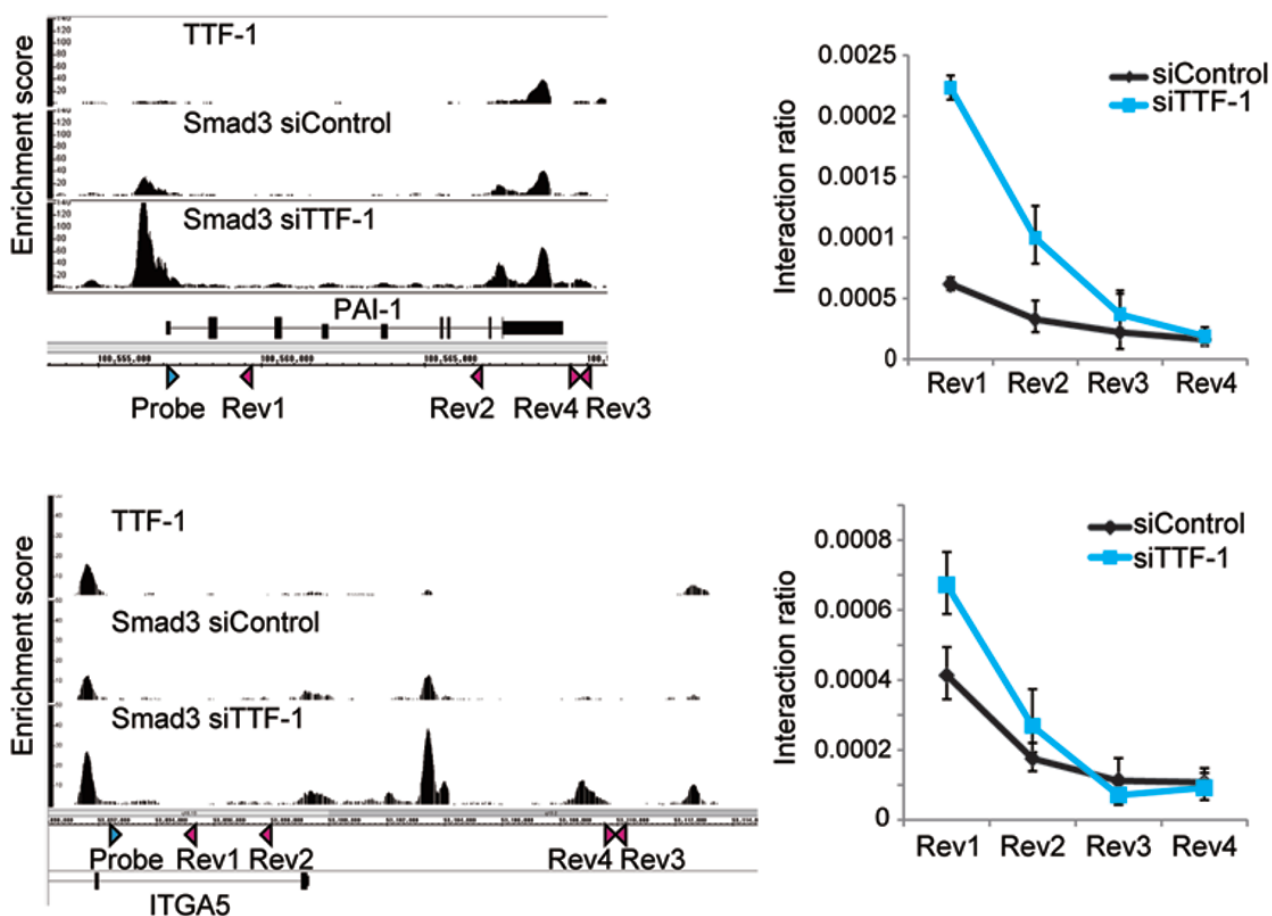
Figure 3 TTF-1 knockdown affects gene expression and genomic interactions at regions with multiple Smad3-binding sits. (A) Comparison of the microarray data of genes induced by TGF- $\beta$ in H441 cells and ChIP-seq data of Smad3. Left column: data from the expression array. Genes expressed in H441 cells and induced by TGF- $\beta$ stimulation are selected and arranged with respect to siTTF-1/siControl ratios (changes in gene expression levels between siTTF-1 and siControl cells). Right column: Smad3 ChIP-seq data. Black bars indicate Smad3 binding with two-fold upregulation by siTTF-1. (B) Gene ontology analysis of the expression microarray data of $\mathrm{H} 441$ cells transfected with siControl or siTTF-1. Effect of the knockdown of TTF-1 on TGF- $\beta$-induced changes in gene expression was evaluated by comparing the enriched gene ontology clusters (left vs right) obtained by DAVID. (C) Gene ontology analysis of the function of TTF-1 in the absence of TGF- $\beta$ stimulation. Acquisition of gene ontology clusters was performed as in B. (D) The 3C assay. Left: if a Smad complex binds to multiple sites in the promoter and assembles different regions after DNA digestion, interaction between multiple binding sites can be detected. Right: if a Smad complex binds to a single site in the promoter and fails to assemble different regions after DNA digestion, interaction between multiple binding sites cannot be observed. (E) Left panels: designed $3 \mathrm{C}$ Taqman probes (blue triangles) and primers (orange triangles) in the PAI-1 and ITGA5 loci. We designed four primers (including one with the opposite direction). The scheme of the PAI-1 promoter (upper left) is a modification of Figure 2D. Right panels: interaction ratios were normalized by input values. Rev1-Rev4 indicate the reverse primers shown in the left panels. Error bars $=$ SD.

ray data using DAVID [30]. We chose genes whose expression levels were increased upon TGF- $\beta$ stimulation in cells transfected with siControl or siTTF-1. Upon TGF- $\beta$ stimulation, only small increases in enrichment scores were seen in the presence of TTF-1, while knockdown of TTF-1 increased expression of many genes, including cell migration- and locomotion-related genes, in addition to genes involved in blood vessel development (Figure 3B). Notably, induction of SNAI1 and SNAI2 by TGF- $\beta$ was enhanced upon knockdown of TTF-1 (Supplementary information, Figure S4B and S4C). Some of the Smad3-binding peaks around these genes represented Smad3-only regions and the binding strengths of Smad3 were strongly affected by TTF-1 expression.

We also analyzed TTF-1-regulated genes in the absence of TGF- $\beta$ by comparing gene expression in siControl and siTTF-1 cells. We chose genes whose expression levels were upregulated in cells transfected with siControl (Figure 3C, left) or siTTF-1 (Figure 3C, right) without TGF- $\beta$ stimulation. We found that TTF-1 up-regulated cell adhesion-related genes, and that knockdown of TTF-1 enhanced expression of apoptosis-related genes. Taken together, these data suggest that TTF-1 suppresses TGF- $\beta$-induced EMT-related genes involved in cell migration and locomotion and protects cells from apoptosis in H441 cells.

Knockdown of TTF-1 enhances the interaction between multiple Smad3-binding regions in Smad3/Smad4 target genes

As shown in Figure 2D and Supplementary information, Figure S4B, we observed multiple Smad3-binding sites around a single target gene locus. Some represent Smad3-TTF-1 common regions, and others are Smad3-only regions. Therefore, it is possible that one Smad complex might bind to multiple sites through the MH1 domains of Smad3 and Smad4 or indirectly through other protein(s); alternatively, each Smad complex may bind to only one SBE. To test this, we performed a chromatin conformation capture (3C) assay. If each Smad protein in the Smad complex binds to the SBE independently and they act together to link distant genomic regions, we can detect interaction(s) of recognition sites of the restriction enzyme between multiple Smad-binding sites (Figure 3D, left). If each Smad complex can bind to only one SBE, such interactions cannot be observed (Figure 3D, right).

We designed probes and primers in two target gene regions, PAI-1 and ITGA5 (Figure 3E). In both regions, a probe was designed immediately downstream of one Smad3-binding site and primers were designed upstream and downstream of other Smad3-binding sites. One of the Smad3-binding sites is common to TTF-1 where Smad3 can bind to chromatin regardless of TTF-1 expression, and the other(s) is Smad3-only region(s) where Smad3-binding strength is weak in the presence of endogenous TTF-1. We detected enhanced interaction using primers upstream of the Smad3-binding sites in cells treated with siTTF-1, and minimal interaction was observed using primers downstream of the Smad3-binding regions. Taken together, these data suggest the existence of interactions between different SBEs in the promoters of PAI-1 and ITGA5 genes, which could be enhanced by TTF-1 knockdown.

Smad3 binds to certain DNA regions with TTF-1 in the absence of TGF- $\beta$ stimulation and regulates target gene expression

Next, we analyzed Smad3-binding regions, which were identified only in cells with siControl but not with siTTF-1 (Figure 2A-i). These Smad3-binding peaks were common to TTF-1 binding, and were therefore 
termed Smad3-TTF-1-requiring loci (STRL). We identified the genes nearest to these loci, and compared their expression in the presence and absence of TGF- $\beta$. Genes located near the STRL, whose expression levels were reduced by more than half after $24 \mathrm{~h}$ of TGF- $\beta$ stimulation, are shown in Supplementary information, Table S8, including $L M O 3$, which exhibits pro-survival effects in adenocarcinoma cells [14]. To determine whether Smad3 binding to the STRL is regulated by TGF- $\beta$ signaling, we performed ChIP-qPCR analysis at these loci in the presence of TGF- $\beta$ or the TGF- $\beta$ type I receptor inhibitor SB431542. Surprisingly, Smad3 bound strongly to these loci in the presence of SB431542, and TGF- $\beta$ treatment reduced the respective binding strengths of Smad3 to these loci (Figure 4A, left). Moreover, knockdown of Smad4 did not attenuate Smad3 binding to these loci (Figure 4A, right), suggesting that Smad3 binds to these loci independently of Smad4. We confirmed colocalization of Smad3 and TTF-1 at the LMO3 region by sequential ChIP using TTF-1 and Smad3 antibodies (Supplementary information, Figure S5). We also found that Smad2 colocalized with TTF-1 at this region, possibly through interaction of Smad2 with Smad3. We examined whether Smad3 or Smad4 affects mRNA expression of these genes, and found that knockdown of either Smad3 or Smad4 cancelled the effects of TGF- $\beta$ (Figure 4B). By knocking down Smad3, the basal expression level of LMO3 was increased, whereas those of SDPR and FBP1 were reduced, suggesting that Smad3 could act as both a repressor and an activator of genes close to the STRL. Expression levels of those genes in A549 cells (which lack endogenous TTF-1 expression) were comparable to those in H441 cells (which express TTF-1) treated with TGF- $\beta$ for $24 \mathrm{~h}$, suggesting that binding of TTF-1-Smad 3 complex to these target regions is important for regulating the expression of these genes in H441 cells.

Finally, we performed Smad3 ChIP-seq analysis using A549 cells treated with TGF- $\beta$, and compared binding regions of Smad3 with those in H441 cells. Approximately $20 \%$ of the Smad3-binding regions were observed at STRL in H441 cells which express TTF-1, while only $1 \%$ of them were observed at STRL in A549 cells (Figure $4 C)$. In A549 cells treated with TGF- $\beta$, Smad3 failed to bind to the STRL at LMO3, SDPR or FBP1 (Supplementary information, Table S9). Read counts of Smad3 were obtained at the Smad3-binding regions in A549 cells treated with TGF- $\beta$ (Supplementary information, Table S9), and compared to those in H441 cells treated with siTTF-1, using scatter plotting (Figure 4D, left). We have also obtained read counts of Smad3 in A549 cells at the STRL in H441 cells, and compared to those in H441 cells treated with siControl (Figure 4D, right).
Correlation of the read counts in Smad3-binding regions was observed between A549 cells and H441 cells treated with siTTF-1 (correlation coefficient $=0.479$ ), while no correlation was observed between A549 cells and H441 cells with siControl in terms of Smad3 binding to the STRL (Figure 4D). These findings suggest that certain portions of Smad3-binding regions in A549 cells are common to those in other lung adenocarcinoma cells, and that Smad3 binding to STRL requires the expression of TTF-1.

\section{Discussion}

Smad4-dependent and-independent functions of Smad3

We demonstrated that TTF-1 binds to Smad3 both in the presence and absence of TGF- $\beta$ stimulation and its presence decreases the amount of Smad3-Smad4 complex in the nucleus (Figure 1A-1D). TTF-1 inhibits both Smad3 and Smad4 binding to chromatin, but in different ways. Smad3 colocalizes with TTF-1 in certain chromatin regions (Figure 2B), while Smad4 was not detected at chromatin in the presence of TTF-1 in the PAI-1 regions (Figure 2D). In addition, Smad4-binding strengths were weaker than those of Smad3 at the Smad3-binding sites where TTF-1 strongly bound, and this tendency was cancelled by knockdown of TTF-1 (Figure 2H). These findings suggest that TTF-1 competes with Smad4 for interaction with Smad3 at the whole Smad-binding sites of the genome.

Our present findings also revealed that Smad3 binds to chromatin with TTF-1, but not with Smad4, in certain regions (Figure 5). Smad3 binds to the promoter regions of $S D P R$ and $F B P 1$, and the second intron of $L M O 3$, where TTF- 1 is also able to bind, in the absence of TGF- $\beta$ signaling. TGF- $\beta$ stimulation reduces the binding strengths of Smad3 at these sites, while knockdown of Smad4 enhanced or did not significantly affect them (Figure 4A). Thus, in cells expressing TTF-1, Smad3 binds to chromatin with TTF-1 without TGF- $\beta$ stimulation, and the Smad3-TTF-1 complex regulates expression of different target genes from those regulated by the Smad3-Smad4 complex.

There are some reports that $\mathrm{Smad} 3$ regulates the expression of target genes in a Smad4-independent manner [31-34]. TRIM33 (also known as TIF1 $\gamma$ and ectodermin) competes with Smad4 for interaction with Smad3 upon TGF- $\beta$ stimulation, and transduces signals independently of Smad4 through the TRIM33/Smad3 complex [33]. Also, SARS-associated coronavirus nucleocapsid protein was reported to interact with Smad3, acting competitively with Smad4, and modulate the expression of target genes of TGF- $\beta$ signaling [34]. In contrast to these cases, 
A ChIP-gPCR
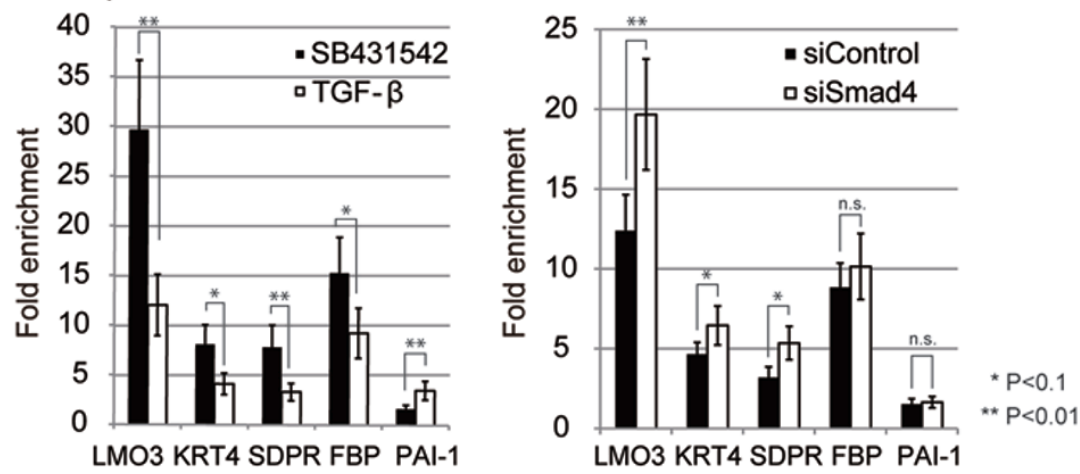

B RT-PCR
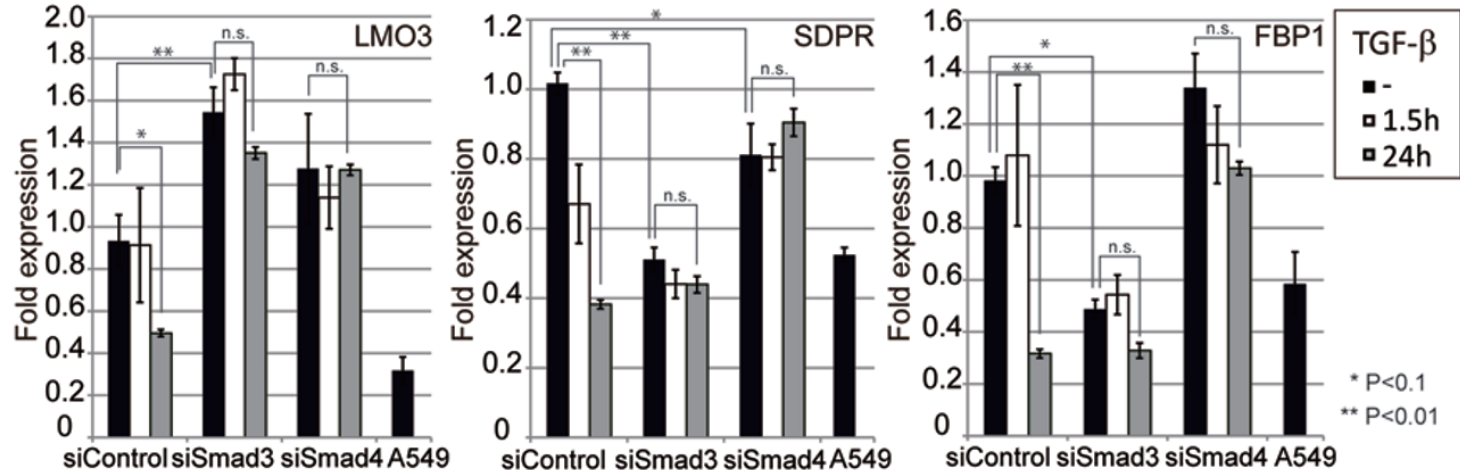

C

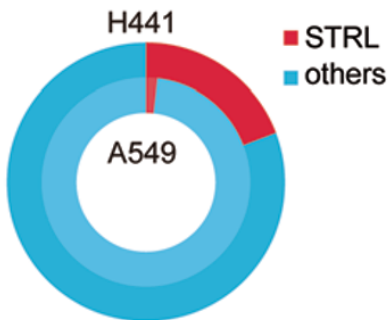

D

Smad3 binding (A549 cells)
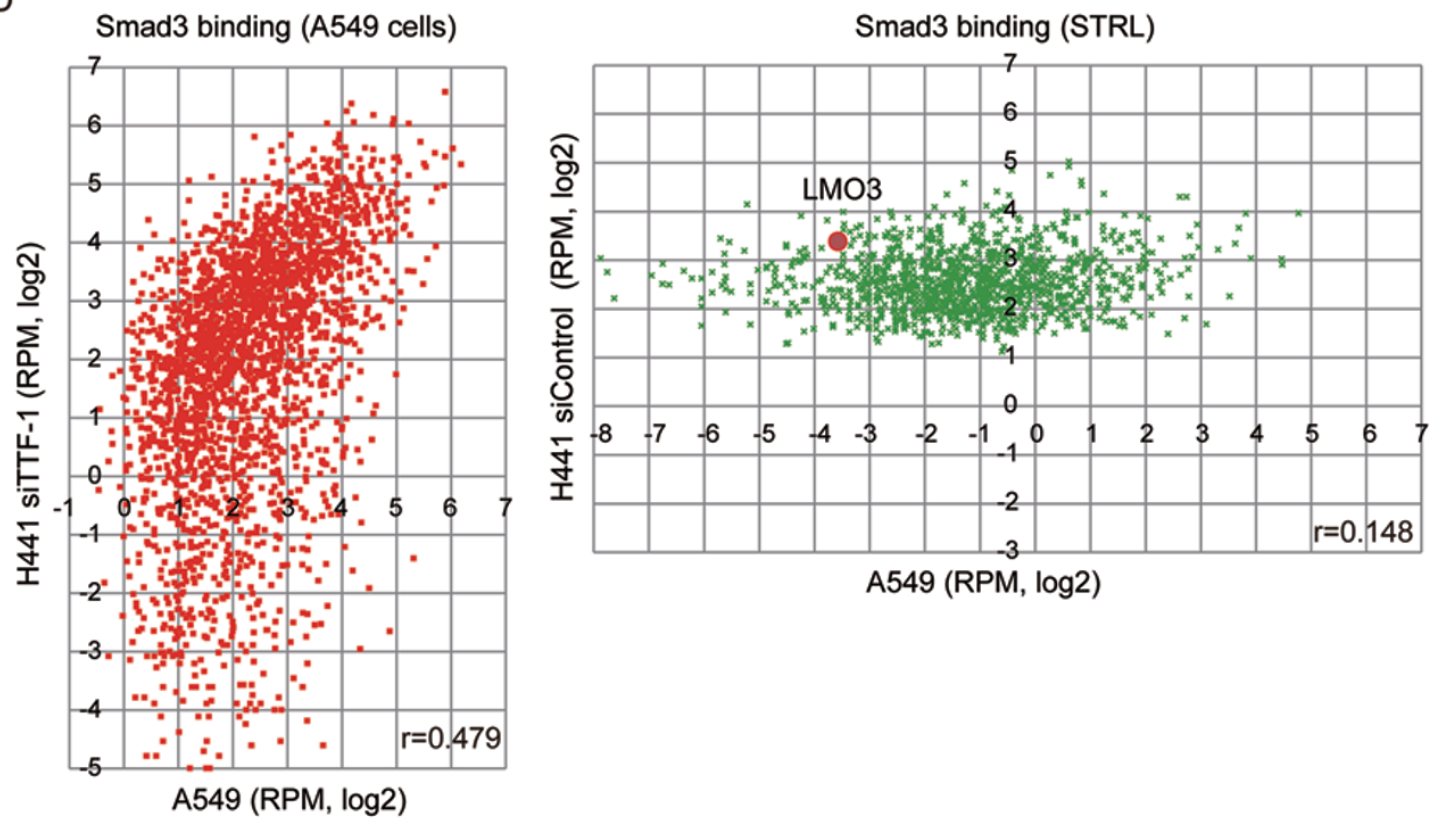

www.cell-research.com | Cell Research 
Figure 4 Smad3 binds to certain chromatin regions with TTF-1 independently of Smad4 in the absence of TGF- $\beta$ stimulation. (A) Left: H441 cells were treated with SB431542 or TGF- $\beta$ for 1.5 h. ChIP-qPCR was performed using Smad3 antibody. Right: H441 cells were treated with control or Smad4 siRNAs for $48 \mathrm{~h}$ without TGF- $\beta$ stimulation, and ChIP-qPCR was performed using Smad3 antibody. LMO3, LIM-domain only-3; KRT4, keratin 4; SDPR, serum deprivation response; and FBP, fructose-1,6-biphosphatase. (B) mRNA expression of target genes near the STRL. H441 cells were treated with control, Smad3, or Smad4 siRNAs for $48 \mathrm{~h}$, and A549 cells were treated with control siRNA for $48 \mathrm{~h}$. H441 cells were stimulated with TGF- $\beta$ as indicated. RT-PCR analyses were performed using primers specific for LMO3 (left), SDPR (middle) or FBP1 (right). (C) Comparison of Smad3-binding loci between A549 cells (inner circle) and H441 cells treated with siControl (outer circle). Red parts show loci which are observed at STRL. (D) Read counts of Smad3 at the Smad3-binding regions in A549 cells were obtained, and comparison was made in each region between A549 cells and H441 cells transfected with siTTF1 (left panel). Read counts of Smad3 at the STRL of H441 cells were also obtained from A549 cells and from H441 cells treated with siControl, and comparison was made in each region (right panel). $r$, Pearson's correlation coefficient; RPM, reads per million mapped reads.

TTF-1 forms complexes with Smad3 without TGF- $\beta$ stimulation. Thus, the Smad4-independent function of Smad3 demonstrated in the present study differs from that induced by the other reported factors.

\section{Mutual inhibition between TGF- $\beta$ and TTF-1 signaling}

The effects of TTF-1 on tumor progression appear to be context dependent [35]. TTF-1 exhibits pro-survival effects through induction of ROR1 and/or LMO3 [13, 14]. In the present study, we have shown that LMO3 expression was regulated by the Smad3-TTF-1 complex in H441 cells. On the other hand, TTF-1 inhibits TGF$\beta$-induced EMT [28], and thus functions as a tumor suppressor gene. TTF-1 may thus have two different effects that are opposite to the effects induced by TGF- $\beta$ signaling: pro-survival and anti-EMT functions. The ontology analysis of the microarray data of $\mathrm{H} 441$ cells by DAVID (Figure 3B and 3C) supports these observations.

TTF-1 regulates specific genes in alveolar epithelial cells, while TGF- $\beta$ induces EMT. Thus, the balance between TTF- 1 expression and TGF- $\beta$ signaling may be very important for differentiation of lung epithelial cells and progression of lung adenocarcinoma. TTF-1 inhibits parts of TGF- $\beta$ signaling, and TGF- $\beta$ conversely inhibits the function of TTF-1. Interaction of Smad3 with TTF1 did not alter the binding profiles of TTF-1 to chromatin (Supplementary information, Figure S6A and S6B). Of note, TGF- $\beta$ reduced the expression of TTF-1 mRNA only at later time points (Supplementary information, Figure S4A), while TTF-1 binding to chromatin was rapidly inhibited by TGF- $\beta$, suggesting that some other mechanisms are important in regulation of the function of TTF- 1 by TGF- $\beta$ signaling.

We previously reported that TTF- 1 inhibits TGF- $\beta$-induced EMT, and suggested that inhibition of the expression of Snail and/or Slug by TTF-1 is important. We also detected up-regulation of SNAII and SNAI2 expression levels (encoding Snail and Slug, respectively) in H441 cells by TGF- $\beta$ (Supplementary information, Figure S4B and S4C). Furthermore, we observed that expression levels of some other genes that are known to be involved in EMT are regulated by TGF- $\beta$ and TTF-1. ITGA5 is reportedly up-regulated during EMT. Moreover, integrin $\alpha 5$ (encoded by ITGA5) interacts with the extracellular matrix and enhances cell migration [36]. Using the $3 \mathrm{C}$ assay, we observed enhanced Smad3 binding to the ITGA5 promoter and the interaction of distant chromatin regions around the ranscription start site in H441 cells upon TTF-1 knockdown. Thus, ITGA5 might be another direct target of TGF- $\beta$, which is suppressed by TTF-1. Also, some genes shown in Supplementary information, Table S7, e.g., $L O X$ and $R U N X 2$, have been reported to promote EMT in some carcinomas $[37,38]$. These genes may play important roles in the progression of malignancy.

\section{Cellular context-dependent function of the TTF-1-Smad3 complex}

In the present study, we have shown that the TTF-1Smad3 complex regulates the expression of genes different from those regulated by the canonical TGF- $\beta$-Smad pathway. In H441 cells, knockdown of TTF-1 enhanced the expression of pro-apoptotic genes in the absence of TGF- $\beta$ stimulation as well as the induction of EMT-related genes upon TGF- $\beta$ stimulation. TTF- 1 inhibits apoptosis of lung epithelial cells, and the TTF-1-Smad3 complex may modulate the expression of apoptosis-related genes. $L M O 3$ is one of such genes regulated by the TTF-1-Smad3 complex, though Smad3 has a suppressive effect on its expression (Figure 4B).

We also obtained RNA-seq data using TTF-1-deficient A549 cells, and performed ontology analysis (Supplementary information, Figure S7A), as in H441 cells (Figure $3 \mathrm{~B}$ and $3 \mathrm{C}$ ). Ectopic expression of TTF-1 inhibited the expression of cell motion-related genes induced by TGF- $\beta$, but did not enhance that of pro-survival-related 
genes, including $L M O 3$ (Supplementary information, Figure S7A). One of the reasons that ectopic TTF-1 failed to upregulate some of its target genes in A549 cells might be due to epigenetic changes. Based on a public database (GEO accession no. GSM999365), CpG methylation was present in the LMO3 locus in A549 cells (data not shown). Moreover, cell growth assay revealed that TTF-1 inhibits the proliferation of A549 cells (Supplementary information, Figure S7B), suggesting that the function of TTF-1 in cell proliferation and apoptosis varies according to cellular contexts, although inhibitory effects of TTF-1 on the Smad3-Smad4 complex and EMT are maintained.

Tissue-specific expression of transcription factors and modification of Smad 3 binding

We propose a hypothesis that each Smad protein in the Smad complex binds to distinct Smad-binding sites, resulting in an assembly connecting multiple promoter and/or enhancer regions of a target gene (Figure 5). Smad3 and Smad4 may also bind to chromatin indirectly

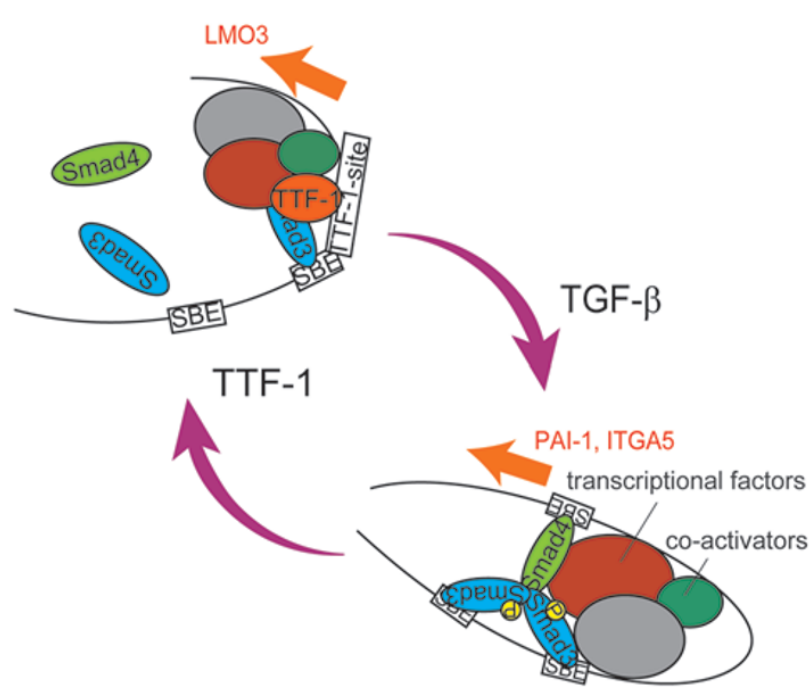

Figure 5 Smad4-independent roles of Smad3 regulated by TTF-1. Scheme of Smad4-dependent and -independent functions of Smad3 in lung adenocarcinoma cells. The Smad3-Smad4 complex interacts with other transcription factors and transcriptional co-activators (or co-repressors), and regulates transcription of certain target genes, including PAI-1 and ITGA5, leading to induction of EMT and other effects upon TGF- $\beta$ stimulation. In the presence of TTF-1, the Smad3-Smad4 complex is disrupted, and TTF-1 interacts with Smad3 and other transcription factors and regulates certain other genes, including $L M O 3$, leading to induction of cell survival and certain other effects in a cellular context-dependent manner. through other proteins. Thus, the increased interaction of promoter and enhancer regions demonstrated in the 3C assay may reflect enhanced binding between Smads and other transcription factors.

Recent studies indicate that co-localization of cell-type-specific transcription factors and $\mathrm{Smad} 3$ is important for context-dependent outputs of TGF- $\beta$ signaling $[19,21]$. We have shown here that TTF-1, a lung epithelial-specific transcription factor, co-occupies with Smad3 on chromatin, and that loss of TTF-1 function greatly alters the binding profiles of Smad3, especially in regions where TTF-1 does not bind to chromatin. These tissue-specific factors seem not only to enable Smad3 to bind to specific regions, but also to inhibit Smad3 binding to certain regions. These factors may bind to unphosphorylated Smad3, as well as to the Smad3-Smad4 complex. Understanding the underlying regulatory mechanisms may help to elucidate the differences in progression and therapeutic response of cancers from different organs.

\section{Materials and Methods}

\section{Cell culture}

A549 cells were maintained in Dulbecco's modified Eagle's medium (\#11965; Life Technologies) supplemented with 10\% fetal bovine serum (FBS), $100 \mathrm{U} / \mathrm{ml}$ penicillin $\mathrm{G}$ and $100 \mu \mathrm{g} / \mathrm{ml}$ streptomycin. H441 cells were maintained in RPMI 1640 (\#11875; Life Technologies) supplemented with $10 \% \mathrm{FBS}, 100 \mathrm{U} / \mathrm{ml}$ penicillin $\mathrm{G}$ and $100 \mu \mathrm{g} / \mathrm{ml}$ streptomycin. Cells were grown in a humidified atmosphere with $5 \% \mathrm{CO}_{2}$ at $37{ }^{\circ} \mathrm{C}$.

\section{Reagents and antibodies}

Recombinant TGF- $\beta$ (TGF- $\beta 3$ ) and the TGF- $\beta$ type I receptor inhibitor SB431542 were purchased from R\&D systems and Sigma-Aldrich (S4317), respectively. The following antibodies were used: mouse anti-FLAG (M2; Sigma-Aldrich), mouse anti-myc (9E10; Oncogene research products), rabbit anti-pSmad3 (C25A9; Cell Signaling), mouse anti-tubulin (DM1A; Sigma-Aldrich), rabbit anti-HDAC1 (2E10; Millipore), mouse anti-Smad2/3 (BD), rabbit anti-Smad3 (ab28379 and ab40854; Abcam), mouse anti-Smad4 (B-8; Santa Cruz), goat anti-Smad4 (AF2097; R\&D) and mouse anti-TTF-1 (8G7G3/1; Novus Biologicals).

\section{Immunoprecipitation and immunoblotting}

Lysis buffer (1\% NP-40, $150 \mathrm{mM} \mathrm{NaCl}, 50 \mathrm{mM}$ Tris- $\mathrm{HCl}(\mathrm{pH}$ 8.0), $5 \mathrm{mM}$ EDTA, $1 \mathrm{mM}$ phenylmethylsulfonyl fluoride and 10 $\mu \mathrm{g} / \mathrm{ml}$ aprotinin) was used for cell lysis. Immunoprecipitation was performed as previously described [39]. Sodium dodecyl sulfate gel electrophoresis and immunoblotting were performed as described [39], using a SEPROS SV (Fujifilm, Japan) or a LAS-4000 lumino-image analyzer (Fujifilm, Japan).

\section{Fractionation of the nucleus and cytoplasm}

We used the NE-PER Nuclear and Cytoplasmic Extraction Kit (Thermo Scientific) according to the manufacturer's instructions. 
We confirmed that the same numbers of cells were applied to the kit by counting cell numbers.

\section{In situ PLA}

We used the Duolink (Olink, Uppsala, Sweden) kit for in situ PLA assay as previously described [40]. Combination of the primary antibodies was determined so that each antibody does not cross-react with the PLA probe-conjugated secondary antibody to the other primary antibodies. TOTO-3 (Invitrogen-Molecular Probes) or Vectashield mounting medium with DAPI (Vector laboratories) was used as a nuclear counterstain.

\section{Promoter-reporter and cDNA constructs}

Human PAI-1 reporter and 9× CAGA reporter were previously described [18]. Human Smad3, Smad4 and TTF-1 cDNAs were prepared as previously reported $[25,28]$. Adenoviral expression vectors of LacZ and TTF-1 were prepared as described [28].

\section{Dual-luciferase assay}

Cells in 24-well plates were transfected with combinations of promoter-reporter constructs and expression plasmids using Lipofectamine LTX (Life Technologies). Total amounts of transfected DNA were adjusted to the same quantities with empty vectors. For normalization, pGL4.75-SV40-hRluc was cotransfected. Twenty-four hours later, cells were stimulated with or without $1 \mathrm{ng} / \mathrm{ml}$ TGF- $\beta$, and then harvested and assayed for luciferase activity $12 \mathrm{~h}$ after stimulation using the Dual-Luciferase Reporter Assay System (Promega). All samples were run in duplicate, and the results were averaged.

\section{RNA interference}

We used the siRNA against human TTF-1 (Invitrogen) as previously reported [28], and control siRNA was purchased from Invitrogen (Cat. 12935-112, sequence not available). siRNAs were introduced into A549 cells twice every $48 \mathrm{~h}$ using Lipofectamine RNAiMAX reagent (Invitrogen) according to the manufacturer's instructions. The final concentration of siRNA in the culture media was $50 \mathrm{nM}$.

\section{Chromatin immunoprecipitation}

A549 cells were cultured in 15 -cm plates to $\sim 80 \%$ confluence, and one plate was used for one immunoprecipitation. H441 cells were cultured in 10-cm plates, and two plates were used for one immunoprecipitation. ChIP was performed as described [21]. ReChIP assay was performed with a similar protocol, but the primary immune complex was eluted by $10 \mathrm{mM} \mathrm{DTT}$ at $37^{\circ} \mathrm{C}$ for $30 \mathrm{~min}$, diluted 50-fold with the ChIP-dilution Buffer as described [21] and immunoprecipitated with secondary antibodies. Fold-enrichment values were calculated by dividing percent input values at target regions by those at the first intron of HPRT1 gene. Primer sequences for ChIP-qPCR are shown in Supplementary information, Data S1.

\section{$R N A$ isolation and $q R T-P C R$}

Total RNAs were extracted as described previously [28]. First-strand cDNAs were synthesized using PrimeScript 2 reverse transcriptase (TakaraBio, Shiga, Japan). qRT-PCR analysis was performed using FastStart Universal SYBR Green Master Mix with ROX (Roche), and the ABI PRISM 7000 Sequence Detec- tion System or the StepONE Plus real time PCR system (Applied Biosystems, CA, USA). All samples were run in triplicate and the results were averaged. Primer sequences for qRT-PCR are shown in Supplementary information, Data S1.

\section{ChIP-seq and data analysis}

High-throughput sequencing of the ChIP fragments was performed using the Illumina Genome Analyzer (Illumina) following the manufacturer's protocols. One flow cell lane was used to sequence each pooled sample. Unfiltered 36-bp sequence reads were aligned against the human reference genome (NCBI Build 36, hg 18) using ELAND (Illumina). Peaks were called using CisGenome v1.2 [41] by two-sample analysis; input genomic DNA was used as a negative control. Assigning a binding site to the nearest gene within $100 \mathrm{~kb}$ of a peak was performed using CisGenome. CisGenome was also used for both de novo motif prediction and motif mapping of TTF-1 ChIP-seq-binding regions.

\section{Expression microarray}

Total RNAs were extracted as described above. The experimental procedures for GeneChip (Affymetrix) were performed as described previously [21] using the GeneChip Human U133 plus 2.0 oligonucleotide arrays (Affymetrix). Microarray Suite software 5.0 (Affymetrix) was used with a target intensity of 100. Data from one array were obtained for each sample. Functional Annotation Clustering function of DAVID 6.7 was used to identify top-enriched clusters by gene ontology analyses.

\section{C assay}

We used Taqman Chromosome Conformation Kits (Life Technologies) for the $3 \mathrm{C}$ assay. $\mathrm{H} 441$ cells were cultured in $10-\mathrm{cm}$ plates, and one plate was used for each 3C assay. EcoRI was used for digestion. qRT-PCR analysis was performed using Taqman gene expression master mix, and a LightCycler 480 (Roche, Switzerland) was used. All samples were run in quintuplicate and the results were averaged. Amplification data were quantified using the $\Delta \Delta C_{\mathrm{t}}$ method (comparative threshold cycle method) and normalized using the human internal control attached to the kit. The sequences of the Taqman probe and primers for 3C assays are shown in Supplementary information, Data S1.

\section{Statistical analysis}

Student's $t$-test was used for two-sample analyses. Bonferroni test of the R program (http://www.r-project.org/) was used for multiple comparisons of the data.

\section{Accession number}

Raw sequencing data with peak calling results and microarray data are available at GEO (GSE51510).

\section{Acknowledgments}

We are grateful to Kaori Shiina, Hiroko Meguro and Keiko Yuki for technical assistance, as well as to members of the Miyazono laboratory for discussion and advice. This research was supported by KAKENHI (grants-in-aid for scientific research) on Innovative Area (Integrative Research on Cancer Microenvironment Network, 22112002 to KM) from the Ministry of Education, Culture, Sports, Science and Technology of Japan (MEXT), and 
Scientific Research (S), 20221009 to HA and Research Activity Start-up, 24890039 to KI from MEXT. This research was also supported by the Ministry of Health, Labor, and Welfare of Japan (a grant to DK), and the Genome Network Project from MEXT (a grant to HA). DK is supported by a grant from Mochida Memorial Foundation for Medical and Pharmaceutical Research and Project for Development of Innovative Research on Cancer Therapeutics from MEXT. The authors declare no competing interests.

\section{References}

1 Boggaram V. Thyroid transcription factor-1 (TTF-1/Nkx2.1/ TITF1) gene regulation in the lung. Clin Sci (Lond) 2009; 116:27-35.

2 Zamecnik J, Kodet R. Value of thyroid transcription factor-1 and surfactant apoprotein $\mathrm{A}$ in the differential diagnosis of pulmonary carcinomas: a study of 109 cases. Virchows Arch 2002; 440:353-361.

3 Moldvay J, Jackel M, Bogos K, et al. The role of TTF-1 in differentiating primary and metastatic lung adenocarcinomas. Pathol Oncol Res 2004; 10:85-88.

4 Saad RS, Liu YL, Han H, Landreneau RJ, Silverman JF. Prognostic significance of thyroid transcription factor-1 expression in both early-stage conventional adenocarcinoma and bronchioloalveolar carcinoma of the lung. Hum Pathol 2004; 35:3-7.

5 Anagnostou VK, Syrigos KN, Bepler G, Homer RJ, Rimm DL. Thyroid transcription factor 1 is an independent prognostic factor for patients with stage I lung adenocarcinoma. $J$ Clin Oncol 2009; 27:271-278.

6 Perner S, Wagner PL, Soltermann A, et al. TTF1 expression in non-small cell lung carcinoma: association with TTF1 gene amplification and improved survival. J Pathol 2009; 217:6572.

7 Winslow MM, Dayton TL, Verhaak RG, et al. Suppression of lung adenocarcinoma progression by Nkx2-1. Nature 2011; 473:101-104.

8 Hosono Y, Yamaguchi T, Mizutani E, et al. MYBPH, a transcriptional target of TTF-1, inhibits ROCK1, and reduces cell motility and metastasis. EMBO J 2012; 31:481-493.

9 Weir BA, Woo MS, Getz G, et al. Characterizing the cancer genome in lung adenocarcinoma. Nature 2007; 450:893-898.

10 Kwei KA, Kim YH, Girard L, et al. Genomic profiling identifies TITF1 as a lineage-specific oncogene amplified in lung cancer. Oncogene 2008; 27:3635-3640.

11 Kendall J, Liu Q, Bakleh A, et al. Oncogenic cooperation and coamplification of developmental transcription factor genes in lung cancer. Proc Natl Acad Sci USA 2007; 104:16663-16668.

12 Tanaka H, Yanagisawa K, Shinjo K, et al. Lineage-specific dependency of lung adenocarcinomas on the lung development regulator TTF-1. Cancer Res 2007; 67:6007-6011.

13 Yamaguchi T, Yanagisawa K, Sugiyama R, et al. NKX2-1/ TITF1/TTF-1-induced ROR1 is required to sustain EGFR survival signaling in lung adenocarcinoma. Cancer Cell 2012; 21:348-361.

14 Watanabe H, Francis JM, Woo MS, et al. Integrated cistromic and expression analysis of amplified NKX2-1 in lung adenocarcinoma identifies LMO3 as a functional transcriptional target. Genes Dev 2013; 27:197-210.
15 Massague J. TGFbeta in cancer. Cell 2008; 134:215-230.

16 Feng XH, Derynck R. Specificity and versatility in TGF-beta signaling through Smads. Annu Rev Cell Dev Biol 2005; 21:659-693.

17 Yagi K, Goto D, Hamamoto T, Takenoshita S, Kato M, Miyazono K. Alternatively spliced variant of Smad2 lacking exon 3. Comparison with wild-type Smad2 and Smad3. J Biol Chem 1999; 274:703-709.

18 Dennler S, Itoh S, Vivien D, ten Dijke P, Huet S, Gauthier JM. Direct binding of Smad3 and Smad4 to critical TGF beta-inducible elements in the promoter of human plasminogen activator inhibitor-type 1 gene. EMBO J 1998; 17:3091-3100.

19 Mullen AC, Orlando DA, Newman JJ, et al. Master transcription factors determine cell-type-specific responses to TGF-beta signaling. Cell 2011; 147:565-576.

20 Morikawa M, Koinuma D, Miyazono K, Heldin CH. Genome-wide mechanisms of Smad binding. Oncogene 2012; 32:1609-1615.

21 Mizutani A, Koinuma D, Tsutsumi S, et al. Cell type-specific target selection by combinatorial binding of $\mathrm{Smad} 2 / 3$ proteins and hepatocyte nuclear factor 4 alpha in HepG2 cells. $J$ Biol Chem 2011; 286:29848-29860.

22 Zhang Y, Handley D, Kaplan T, et al. High throughput determination of TGFbeta1/SMAD3 targets in A549 lung epithelial cells. PLoS One 2011; 6:e20319.

23 Kim SW, Yoon SJ, Chuong E, et al. Chromatin and transcriptional signatures for Nodal signaling during endoderm formation in hESCs. Dev Biol 2011; 357:492-504.

24 Brown S, Teo A, Pauklin S, et al. Activin/Nodal signaling controls divergent transcriptional networks in human embryonic stem cells and in endoderm progenitors. Stem Cells 2011; 29:1176-1185.

25 Koinuma D, Tsutsumi S, Kamimura N, et al. Chromatin immunoprecipitation on microarray analysis of Smad2/3 binding sites reveals roles of ETS1 and TFAP2A in transforming growth factor beta signaling. Mol Cell Biol 2009; 29:172-186.

26 Li C, Zhu NL, Tan RC, Ballard PL, Derynck R, Minoo P. Transforming growth factor-beta inhibits pulmonary surfactant protein B gene transcription through SMAD3 interactions with NKX2.1 and HNF-3 transcription factors. $J$ Biol Chem 2002; 277:38399-38408.

27 Minoo P, Hu L, Zhu N, et al. SMAD3 prevents binding of NKX2.1 and FOXA1 to the SpB promoter through its MH1 and MH2 domains. Nucleic Acids Res 2008; 36:179-188.

28 Saito RA, Watabe T, Horiguchi K, et al. Thyroid transcription factor-1 inhibits transforming growth factor-beta-mediated epithelial-to-mesenchymal transition in lung adenocarcinoma cells. Cancer Res 2009; 69:2783-2791.

29 Tagne JB, Gupta S, Gower AC, et al. Genome-wide analyses of Nkx2-1 binding to transcriptional target genes uncover novel regulatory patterns conserved in lung development and tumors. PLoS One 2012; 7:e29907.

30 Dennis G Jr, Sherman BT, Hosack DA, et al. DAVID: Database for Annotation, Visualization, and Integrated Discovery. Genome Biol 2003; 4:P3.

31 Levy L, Hill CS. Smad4 dependency defines two classes of transforming growth factor-beta (TGF-beta) target genes and distinguishes TGF-beta-induced epithelial-mesenchymal transition from its antiproliferative and migratory responses. $\mathrm{Mol}$ 
Cell Biol 2005; 25:8108-8125.

32 Ijichi H, Otsuka M, Tateishi K, et al. Smad4-independent regulation of $\mathrm{p} 21 / \mathrm{WAF} 1$ by transforming growth factor-beta. Oncogene 2004; 23:1043-1051.

33 He W, Dorn DC, Erdjument-Bromage H, Tempst P, Moore MA, Massague J. Hematopoiesis controlled by distinct TIF1gamma and Smad4 branches of the TGFbeta pathway. Cell 2006; 125:929-941.

34 Zhao X, Nicholls JM, Chen YG. Severe acute respiratory syndrome-associated coronavirus nucleocapsid protein interacts with Smad3 and modulates transforming growth factor-beta signaling. J Biol Chem 2008; 283:3272-3280.

35 Yamaguchi T, Hosono Y, Yanagisawa K, Takahashi T. NKX21/TTF-1: An enigmatic oncogene that functions as a double-edged sword for cancer cell survival and progression. Cancer Cell 2013; 23:718-723.

36 Maschler S, Wirl G, Spring H, et al. Tumor cell invasiveness correlates with changes in integrin expression and localization. Oncogene 2005; 24:2032-2041.
37 Taylor MA, Amin JD, Kirschmann DA, Schiemann WP. Lysyl oxidase contributes to mechanotransduction-mediated regulation of transforming growth factor-beta signaling in breast cancer cells. Neoplasia 2011; 13:406-418.

38 Niu DF, Kondo T, Nakazawa T, et al. Transcription factor Runx2 is a regulator of epithelial-mesenchymal transition and invasion in thyroid carcinomas. Lab Invest 2012; 92:11811190.

39 Koinuma D, Shinozaki M, Nagano Y, et al. RB1CC1 protein positively regulates transforming growth factor-beta signaling through the modulation of Arkadia E3 ubiquitin ligase activity. J Biol Chem 2011; 286:32502-32512.

40 Yamazaki T, Yoshimatsu Y, Morishita Y, Miyazono K, Watabe T. COUP-TFII regulates the functions of Prox 1 in lymphatic endothelial cells through direct interaction. Genes Cells 2009; 14:425-434.

41 Ji H, Jiang H, Ma W, Johnson DS, Myers RM, Wong WH. An integrated software system for analyzing ChIP-chip and ChIPseq data. Nat Biotechnol 2008; 26:1293-1300.

(Supplementary information is linked to the online version of the paper on the Cell Research website.) 